\title{
Screening for coronary artery disease in diabetic patients: A commentary
}

\author{
Daniel S. Berman, MD, ${ }^{\mathrm{a}, \mathrm{b}}$ Alan Rozanski, MD, Jamal S. Rana, MD, PhD, ${ }^{\mathrm{a}, \mathrm{b}}$ \\ Leslee J. Shaw, PhD, ${ }^{d}$ Nathan D. Wong, PhD, ${ }^{e}$ and James K. Min, MD ${ }^{f}$
}

Diabetes is a major coronary artery disease (CAD) risk factor which is increasing in prevalence, and commonly associated with asymptomatic CAD. ${ }^{1}$ Patients with diabetes without previous myocardial infarction ${ }^{2}$ or cardiovascular disease ${ }^{3}$ have been shown to have a similar prognosis as persons without diabetes but with prior myocardial infarction or cardiovascular disease. ${ }^{2}$ Based on these group and population-based data, diabetes is considered a "CAD risk-equivalent" according to current Framingham Risk Score Adult Treatment Program III guidelines, and secondary prevention of CAD is recommended for all adult diabetic patients. ${ }^{3}$

However, to date, what has been unresolved is whether there is additional clinical benefit for routine screening of individual asymptomatic diabetics for CAD. In this regard, the recently reported long-term follow-up from the Diagnostic Imaging in Asymptomatic Diabetics (DIAD) trial represents an important first step forward toward answering this question. ${ }^{4}$ The DIAD study examined whether screening of diabetic patients with SPECT myocardial perfusion imaging (MPI) could enhance detection of those with high CAD risk and whether the detection of this CAD risk was associated with an improvement in clinical outcomes.

From the Departments of Imaging and Medicine and the Burns and Allen Research Institute, ${ }^{\mathrm{a}}$ Cedars-Sinai Medical Center, Los Angeles, CA; Department of Medicine, ${ }^{\mathrm{b}}$ David Geffen School of Medicine, University of California, Los Angeles, CA; Division of Cardiology, ${ }^{c}$ St. Lukeós Roosevelt Hospital, New York, NY; Department of Medicine, ${ }^{\mathrm{d}}$ Emory University School of Medicine, Atlanta, GA; Heart Disease Prevention Program, Department of Medicine, ${ }^{\mathrm{e}}$ University of California, Irvine, CA; and Departments of Medicine and Radiology, ${ }^{\mathrm{f}}$ Weill Cornell Medical College and the New York Presbyterian Hospital, New York, NY.

Supported in part by a grant from The Eisner Foundation.

Reprint requests: Daniel S. Berman, MD, Departments of Imaging and Medicine and the Burns and Allen Research Institute, Cedars-Sinai Medical Center, 8700 Beverly Blvd, Room 1258, Los Angeles, CA 90048; bermand@cshs.org.

J Nucl Cardiol 2009;16:851-4.

$1071-3581 / \$ 34.00$

Copyright (C) 2009 by the The Author(s). This article is published with open access at Springerlink.com.

doi:10.1007/s12350-009-9129-2
In 1123 asymptomatic individuals with type 2 diabetes, SPECT-MPI screening was compared to usual care. While rates of hard cardiac events were low in both the SPECT-MPI $(2.7 \%)$ and the usual care $(3.0 \%)$ groups, SPECT-MPI was nevertheless able to successfully discriminate those with higher likelihood of cardiac events based upon moderate or large perfusion deficits versus those with a very low likelihood of future cardiac events based upon normal perfusion or small perfusion deficits ( $2.4 \%$ vs. $0.4 \%, P=0.001)$. Despite this discriminatory ability, however, SPECT-MPI screening was not associated with an improvement in cardiac outcomes, and the DIAD investigators concluded that " routine screening for inducible ischemia in asymptomatic patients with type 2 diabetes cannot be advocated."

Although we are in agreement with this general conclusion, we propose below an important role for SPECT-MPI for the selective evaluation of asymptomatic patients with diabetes as well as an alternative paradigm for more effective CAD screening. Numerous prior studies comparing individuals with and without diabetes have demonstrated that diabetes is associated with heightened mortality among patients with ischemic SPECT-MPI stratified according to any level of myocardial ischemia., ${ }^{5,6}$ Within the DIAD trial, there was similarly a stepwise increase in cumulative cardiac events among those with increasing SPECT-MPI abnormality, paralleling prior experience. In fact, among patients with at least moderate ischemia, the hard cardiac event rate was $12 \%$ vs. $2 \%$ in the normal scan group. Notably, however, only $33(8 \%)$ of the 561 patients within the scan group had a moderate perfusion defect or greater, making this a trial of primarily nonischemic patients. Importantly, this low frequency of ischemia may help explain why the annualized cardiac event rate was only $0.6 \%$ per year in the DIAD study, thus making it difficult to discern meaningful differences within the SPECT-MPI versus usual care arm of this trial.

Still, as aforementioned, in contrast to the results of the current DIAD trial, prior results have suggested that nonischemic SPECT-MPI patients with diabetes are an intermediate risk group for clinical events. One likely explanation is the temporal difference in these follow-up studies, with the contemporary DIAD study coinciding 
with a time of increasingly aggressive medical treatment of diabetes and other CAD risk factors. In support of this hypothesis is the notable finding within the DIAD study of marked increase from baseline to 5 years for use of aspirin, diabetes control, lipid lowering, and antihypertensive therapies within both the scan and no-scan groups. This considerable intensification in medication use was undoubtedly influential in both lowering mortality rates and diluting potential clinical differences within the two arms of the DIAD trial. Prior studies of patients tested in an era predating this type of aggressive medical therapy may have manifested higher clinical event rates among nonischemic SPECT MPI patients.

As current medical therapy is reducing the risk of cardiac events in the average asymptomatic diabetic patient, adjustments are now necessary to redefine a sufficiently high-risk asymptomatic diabetic subgroup that will benefit from stress imaging for ischemia. In this regard, the application of coronary artery calcium (CAC) scanning by noncontrast computed tomography (CT) may represent an important vehicle by which to achieve this objective. ${ }^{7}$ CT coronary calcium scanning is an inexpensive test with no injections and very low radiation exposure. Since CAC is almost exclusively a marker of atherosclerosis, its assessment provides information regarding the extent of coronary plaque burden. A substantial body of evidence has definitively established that the Agatston coronary calcium score (CCS) - a widely used measure of the extent of CAC - provides risk stratification in the asymptomatic general population, ${ }^{8-12}$ with superiority to other imaging screening tests, such as carotid intima-medial thickness scanning. ${ }^{13}$

\section{No Diabetes $(n=9,474)$}

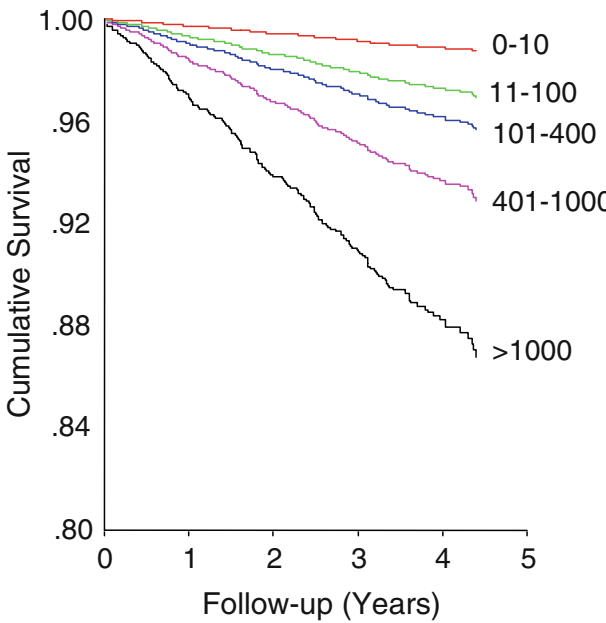

Specific to the assessment of diabetes, CAC measurement provides potent risk stratification for asymptomatic diabetics. In a large study of 10,377 patients (903 diabetics, age $57 \pm 10$ years) who underwent CAC scanning and were then followed for 5 years for all-cause mortality, Raggi et al found that for any category of CAC over zero, there was a stepwise increased mortality risk in individual with diabetes compared to those without, as shown in Figure 1. ${ }^{14}$ Notwithstanding the ability of CAC to predict incident cardiac risk, the converse also appears to hold true; namely, individuals with diabetes with a low CAC score have a very favorable prognosis. Within this study, among the $39 \%$ of diabetics with a CAC score of $<10$, the 5-year all-cause death rate was extremely low, and was similar to rates for those without diabetes with similar CAC scores.

The recently conducted PREDICT Study was a prospective cohort study of 589 asymptomatic type 2 diabetics (median age 63.1) with no history of cardiovascular disease, designed to evaluate $\mathrm{CAC}$ as a predictor of cardiovascular events. CAC was a highly significant, independent predictor of events $(P<0.001)$, with a doubling in CAC being associated with a $29 \%$ increase in risk of events. ${ }^{9} \mathrm{CAC}$ provided greater predictive value for cardiac events than Framingham Risk Score and UKPDS risk scores as well as to conventional and novel risk factors. Of note, $23 \%$ of the patients had CAC scores $\leq 10$, despite the older age of the population than in most other reports, and these patients had a $0.2 \%$ event rate per year.

In a related study, Anand et al ${ }^{15}$ reported CAC scores $<10$ to be present in more than $50 \%$ of individuals with diabetes (age $52 \pm 8$ years) reflecting values similar to

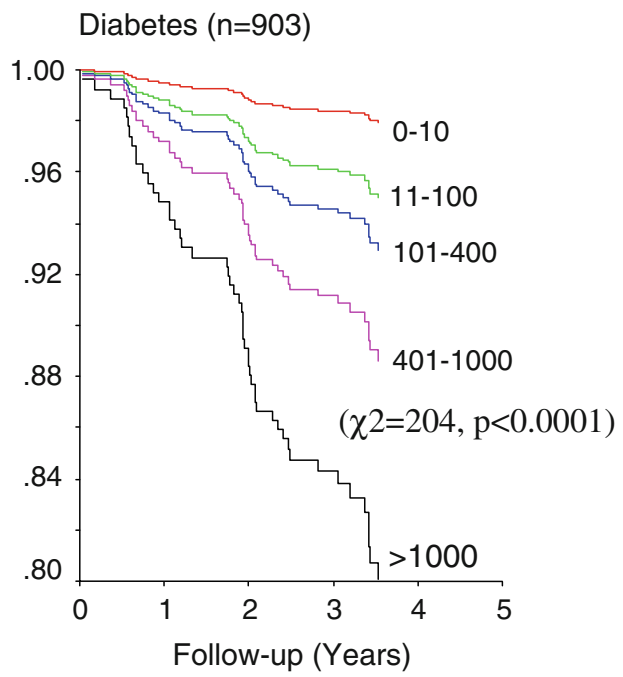

Figure 1. Cox proportional hazards survival in 10,377 patients undergoing coronary artery calcium scanning were followed up $5.0 \pm 3.5$ years for all-cause mortality. Overall, the death rate was $3.5 \%$ and $2.0 \%$ for individuals with diabetes and without diabetes $(P<0.0001)$. (Adapted with permission from J Am Coll Cardiol. ${ }^{14}$ ). 
those derived from our laboratory in over 1000 adult diabetics which has shown that $30 \%$ have a CCS of 0 and $8 \%$ have a CCS of 0-9 (unpublished data). We have also published data showing a fourth of men and nearly half of women with diabetes have no detectable coronary calcium. ${ }^{15}$ This finding could be of major psychological import to such patients, and may have favorable implications for the downstream use of medical resource utilization (e.g., clinical visits and frequency of subsequent stress testing). A prospective study is needed to test this possibility.

Among patients who do have CAC, we and others have also demonstrated a threshold relationship between the magnitude of CAC and the likelihood of observing inducible myocardial ischemia. Within general populations-including our own-this threshold has been observed to be a CAC score $\geq 400$. When limited to asymptomatic nondiabetic patients, we have noted that a CAC score threshold of 1000 may be more robust for discriminating those individuals with a low frequency of inducible ischemia (unpublished observations). Based upon the extrapolation of data from stress testing, the CAC threshold of 400 appears appropriate for asymptomatic individuals with diabetes compared to those without diabetes. ${ }^{16}$ The data from Anand et $\mathrm{al}^{15}$ also support a CAC score of 400, the threshold for identifying sufficiently high CAD risk as to warrant SPECT-MPI in asymptomatic diabetics.

A recent consensus statement by Bax et al has formulated a strategy which purports to improve the potential cost-effectiveness of screening using CAC scanning by selectively employing it among those with high-risk clinical features including other atherosclerotic vascular disease, microalbuminuria and chronic kidney disease, abnormal resting ECG, retinopathy, hyperglycemia, multiple other cardiac risk factors, and age over 65 years. ${ }^{17} \mathrm{~A}$ further study in this regard by Mazzone et al reported in 439 asymptomatic individuals with diabetes that age, systolic blood pressure, male gender, and triglyceride-rich lipoproteins are predictors of CAC in diabetics. ${ }^{18}$ In their study, measures of adipose tissue distribution were significantly associated with CAC score, whereas inflammatory and metabolic factors were not. Further study is needed to evaluate which clinical data may be most useful in tailoring the application of CAC scanning among asymptomatic diabetics.

At the time that the DIAD study was conceived and initiated, the relationship between $\mathrm{CAC}$ scan results and SPECT-MPI findings as well as the utility of using CAC scanning for risk stratification in asymptomatic individuals with diabetes was not known. These data were introduced during the follow-up phase of the DIAD trial. By employing these contemporary findings, it is now possible to test a new paradigm for screening asymptomatic patients with diabetes, the rationale for which has recently been explored in a comprehensive editorial by Dr. Beller. ${ }^{19}$ This paradigm calls for using CAC scanning rather than cardiac stress testing as the first-line screening test among such diabetics. It is expected that approximately one-third of such patients would have no detectable CAC, and many others would have CAC scores at levels which obviate the need for SPECT-MPI. Above a certain CAC threshold (e.g., 400 or possibly 100), SPECT-MPI could be selectively employed as a means for identifying silent ischemia and elevated clinical risk. Conceivably, the inclusion of various clinical parameters such as those suggested by Bax et $\mathrm{al}^{17}$ could alter the threshold criteria used to guide referral to stress testing among diabetic patients (i.e., selective use of a lower CAC threshold among those with high-risk clinical features). When high-risk ischemia is present, ${ }^{20,21}$ coronary angiography would be recommended, based on findings that this appears to be a group who benefit from revascularization. ${ }^{20}$ In patients with $<10 \%$ ischemia, repeat MPI might be appropriate in 3 years. $^{22}$ While it might be of interest to determine the added value of noninvasive coronary CT angiography over CAC scanning in patients with diabetes, ${ }^{22}$ such consideration awaits the publication of evidence that this added testing would provide sufficient additional value to be cost-effective.

A major problem with this approach has been that CAC scanning has not been widely covered by insurance carriers, generally making it available only in research studies or to patients who can afford to pay for the studies on their own. It is noteworthy in this regard that the Texas Legislature has passed legislation requiring insurance plans to provide coverage for screening with the CAC or carotid IMT scans. This coverage requirement applies to men ages 46 to 75 and women ages 56 to 75 who are either diabetic or have an intermediate or higher risk for developing heart disease. ${ }^{23}$

In all likelihood, the most of the small number of patients who had moderate to severe ischemia within the DIAD study would have been identified as deserving selective referral for SPECT-MPI according to this paradigm, based on prior observation regarding the relationship of CAC scores among ischemic SPECT-MPI patients. ${ }^{24}$ Many other patients within the DIAD trial, however, would have been selectively referred away from SPECT-MPI imaging, but with their burden of underlying atherosclerosis and thus long-term risk, better characterized by virtue of having undergone CAC scanning. Given the growing threat posed by the increasing prevalence of diabetes, testing of algorithms which cost-effectively select for the identification of high-risk asymptomatic individuals with diabetes is urgently warranted and not negated by the results of the DIAD trial. 


\section{Open Access}

This article is distributed under the terms of the Creative Commons Attribution Noncommercial License which permits any noncommercial use, distribution, and reproduction in any medium, provided the original author(s) and source are credited.

\section{References}

1. Beckman JA, Creager MA, Libby P. Diabetes and atherosclerosis: Epidemiology, pathophysiology, and management. JAMA 2002;287:2570-81.

2. Haffner SM, Lehto S, Ronnemaa T, Pyorala K, Laakso M. Mortality from coronary heart disease in subjects with type 2 diabetes and in nondiabetic subjects with and without prior myocardial infarction. N Engl J Med 1998;339:229-34.

3. http://www.nhlbi.nih.gov/guidelines/cholesterol/atp3_rpt.htm.

4. Young LH, Wackers FJ, Chyun DA, et al. Cardiac outcomes after screening for asymptomatic coronary artery disease in patients with type 2 diabetes: The DIAD study: A randomized controlled trial. JAMA 2009;301:1547-55.

5. Kang X, Berman DS, Lewin $\mathrm{H}$, et al. Comparative ability of myocardial perfusion single-photon emission computed tomography to detect coronary artery disease in patients with and without diabetes mellitus. Am Heart J 1999;137:949-57.

6. Giri S, Shaw LJ, Murthy DR, et al. Impact of diabetes on the risk stratification using stress single-photon emission computed tomography myocardial perfusion imaging in patients with symptoms suggestive of coronary artery disease. Circulation 2002; 105:32-40.

7. Rumberger JA. Clinical use of coronary calcium scanning with computed tomography. Cardiol Clin 2003;21:535-47.

8. Detrano R, Guerci AD, Carr JJ, et al. Coronary calcium as a predictor of coronary events in four racial or ethnic groups. N Engl J Med 2008;358:1336-45

9. Budoff MJ, Shaw LJ, Liu ST, et al. Long-term prognosis associated with coronary calcification: observations from a registry of 25,253 patients. J Am Coll Cardiol 2007;49:1860-70.

10. Becker A, Leber A, Becker C, Knez A. Predictive value of coronary calcifications for future cardiac events in asymptomatic individuals. Am Heart J 2008;155:154-60.

11. Sarwar A, Shaw LJ, Shapiro MD, et al. Diagnostic and prognostic value of absence of coronary artery calcification. JACC Cardiovasc Imaging 2009;2:675-88

12. Blaha M, Budoff MJ, Shaw LJ, et al. Absence of coronary artery calcification and all-cause mortality. JACC Cardiovasc Imaging 2009;2:692-700
13. Folsom AR, Kronmal RA, Detrano RC, et al. Coronary artery calcification compared with carotid intima-media thickness in the prediction of cardiovascular disease incidence: The Multi-Ethnic Study of Atherosclerosis (MESA). Arch Intern Med 2008;168: 1333-9.

14. Raggi P, Shaw LJ, Berman DS, Callister TQ. Prognostic value of coronary artery calcium screening in subjects with and without diabetes. J Am Coll Cardiol 2004;43:1663-9.

15. Anand DV, Lim E, Darko D, et al. Determinants of progression of coronary artery calcification in type 2 diabetes role of glycemic control and inflammatory/vascular calcification markers. J Am Coll Cardiol 2007;50:2218-25.

16. Wong ND, Rozanski A, Gransar H, et al. Metabolic syndrome and diabetes are associated with an increased likelihood of inducible myocardial ischemia among patients with subclinical atherosclerosis. Diabetes Care 2005;28:1445-50.

17. Bax JJ, Young LH, Frye RL, Bonow RO, Steinberg HO, Barrett EJ. Screening for coronary artery disease in patients with diabetes. Diabetes Care 2007;30:2729-36.

18. Mazzone T, Meyer PM, Kondos GT, et al. Relationship of traditional and nontraditional cardiovascular risk factors to coronary artery calcium in type 2 diabetes. Diabetes 2007;56:849-55.

19. Beller GA. Noninvasive screening for coronary atherosclerosis and silent ischemia in asymptomatic type 2 diabetic patients: Is it appropriate and cost-effective? J Am Coll Cardiol 2007;49:1918-23.

20. Hachamovitch R, Hayes SW, Friedman JD, Cohen I, Berman DS. Comparison of the short-term survival benefit associated with revascularization compared with medical therapy in patients with no prior coronary artery disease undergoing stress myocardial perfusion single photon emission computed tomography. Circulation 2003;107:2900-7.

21. Shaw LJ, Berman DS, Maron DJ, et al. Optimal medical therapy with or without percutaneous coronary intervention to reduce ischemic burden: Results from the Clinical Outcomes Utilizing Revascularization and Aggressive Drug Evaluation (COURAGE) trial nuclear substudy. Circulation 2008;117:1283-91.

22. Hendel RC, Berman DS, Di Carli MF, et al. Appropriate use criteria for cardiac radionuclide imaging. A Report of the American College of Cardiology Foundation Appropriate Use Criteria Task Force, the American Society of Nuclear Cardiology, the American College of Radiology, the American Heart Association, the American Society of Echocardiography, the Society of Cardiovascular Computed Tomography, the Society for Cardiovascular Magnetic Resonance, and the Society of Nuclear Medicine. Circulation 2009;119:e561-87.

23. Texas HB 1290, June 2009. http://www.legis.state.tx.us/billlookup/ BillStages.aspx ?LegSess=81R \&Bill=HB120.

24. Berman DS, Wong ND, Gransar H, et al. Relationship between stress-induced myocardial ischemia and atherosclerosis measured by coronary calcium tomography. J Am Coll Cardiol 2004;44:92330 . 